indexecomunicación | no 9 (3) 2019 | Páginas 59-74

E-ISSN: 2174-1859 | ISSN: 2444-3239 | Depósito Legal: M-19965-2015

Recibido el 31_08_2019 | Aceptado el 12_10_2019 | Publicado el 16_11_2019

\title{
BREVE HISTORIA DE LA TELEVISIÓN TALIANA DESTINADA A INMIGRANTES: EL CASO DE BABEL TV
}

ASHORT HISTORY OF TALIAN TELEVISION AIMED AT IMMIGRANTS:

THECASEOFBABEL TV

\section{Gaetana Cava}

gaetanacava@correo.ugr.es (iD https://orcid.org/0000-0002-7358-2393

Universidad de Granada

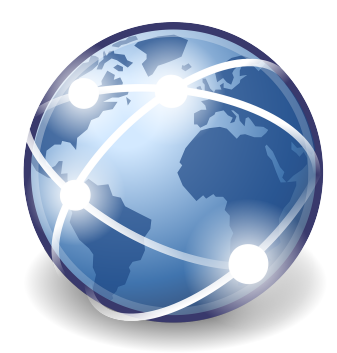

Para citar este trabajo: Cava, G. (2019). Breve historia de la televisión italiana destinada a inmigrantes: el caso de Babel Tv. index.comunicación, 9(3), 59-74. 
Resumen: En los países con una más antigua historia de inmigración con respecto a Italia, los medios de comunicación administrados por extranjeros son una realidad consolidada. En este trabajo, se presentarán la variedad de experiencias televisivas italianas que en particular se han destinado a ciudadanos inmigrantes. Se analizará, además, con mayor enfoque la oferta televisiva de una programación italiana diseñada «a medida del inmigrante»: Babel TV (canal Sky creado en 2010 y que se emitió hasta 2014). En la segunda parte del artículo se examinará el visionado televisivo de este canal por parte de los inmigrantes. La investigación empírica se ha realizado en la ciudad de Messina, con entrevistas a hombres y mujeres de las tres nacionalidades extranjeras más numerosas en dicha ciudad: srilankeses, filipinos y chinos (ANPAL, 2017). Nuestro análisis intentará incluir los siguientes aspectos: fidelización de los sujetos a este canal, preferencias entre las tipologías propuestas por Babel TV y el papel que este espacio televisivo ha desarrollado en mantener una relación entre país de origen y país de destino. Se concluye que los inmigrantes realizan el consumo televisivo a partir de sus necesidades y de acuerdo con factores sociales, culturales y económicos que conforman las identidades individuales y sociales. Palabras clave: televisión; audiencia; narración; inmigrantes; imaginario social.

Abstract: In those countries where immigration has more ancient roots than in Italy, media run by foreigners are a consolidated reality. In this contribution, we will present the media framework that Italian broadcasting, from its origins to the present day, has specifically dedicated to immigrant audiences. In particular, we will analyse the case study of an Italian television service designed for immigrants: Babel TV (a Sky channel that run from 2010 to 2014). In the second part of the article, we will examine the television consumption of this channel by immigrants. The empirical research was carried out in the city of Messina by means of interviews with men and women belonging to the three most numerous foreign communities in the town: Sri Lankan, Filipino and Chinese (ANPAL, 2017). In our analysis we will try to understand the following aspects: the loyalty of customers to this television channel, their preferences among the various genres proposed by Babel TV, and the role that this television airtime has had in maintaining a relationship between their country of origin and the host country. It is concluded that the immigrants consume television on behalf of their needs and according to social, cultural and economic factors, which conform the individual and social identities. Keywords: Television; Audience; Storytelling; Immigrants; Social Imaginary. 


\section{Introducción}

El estudio de este artículo se enmarca en una investigación más amplia acerca del papel que los medios de comunicación italianos, especialmente la televisión, desempeñan dentro de los procesos de integración/exclusión de los inmigrantes (Bramanti, 2011; Sciortino, 2015). Nuestro trabajo propone investigar las transformaciones culturales en relación con el fenómeno migratorio (Allievi, 2019). Sobre todo, es preciso analizar el cambio de los consumos mediáticos como potencial clave de lectura (Pedroza, 2004).

Por más que la investigación empírica de nuestros trabajos esté enfocada en el consumo televisivo por parte de los inmigrantes, en este artículo, en primer lugar, es importante reconstruir el marco mediático que las emisoras italianas, desde su origen hasta nuestros días, han dedicado específicamente a la audiencia inmigrante. Por lo tanto, se pasará revista brevemente a los programas dirigidos en particular a ese tipo de público. Como se observará a lo largo de este artículo, la escasa longevidad de esos programas delata cierto atraso en los medios de comunicación italianos respecto a los procesos interculturales. En los países con una historia de inmigración más antigua que la de Italia, los medios gestionados por extranjeros son una realidad consolidada ${ }^{1}$ (Musarò y Parmiggiani, 2014); el caso italiano enseña un vacío narrativo especialmente por parte de los medios de comunicación mainstream. La literatura científica italiana se ha dedicado de manera predominante a estudiar la representación televisiva de los inmigrantes (Baggiani, Longoni y Solano, 2011; Balma Tivola, 2001; Binotto, Bruno y Lai, 2016; Binotto y Martino, 2004; Calvanese, 2011; Carzo, 2011; Censis, 2002; Corsini, 2013; Eberl et al., 2018; Guadagnucci, 2010; Igartua, Barrios y Ortega, 2012; Maneri, 2001; Marcos Ramos, González de Garay y Portillo Delgado, 2019); pero son escasos los estudios enfocados a analizar la producción de los medios de comunicación que la literatura especializada denomina 'étnicos'2 (Maneri, 2011; Meli, 2011; Repoll, 2004; Vittadini, 2007) $\mathrm{y}$, en general, en los inmigrantes como audiencias (AA.VV., 2012; Altarriba, 2008; Belluati, 2015; Bonerba, 2013; Carzo 2016; Gadotti, 2009; Istat, 2008; King y Wood, 2013; Navarro, 2015; Vittadini, 2008).

Después de esta introducción, que traza el perímetro de nuestro estudio, el artículo se divide en las siguientes secciones: en primer lugar, se detallará la metodología utilizada; luego, se describirán los espacios de la programación italiana que específicamente se han destinado a ciudadanos inmigrantes. Se empezará por el servicio público italiano para, a continuación, dirigir la atención a las emisoras privadas y, finalmente, concluir con un análisis pormenorizado de Babel TV, del canal Sky, creado en 2010 y que se emitió hasta 2014. A continuación, presentaremos el corpus de noticias seleccionado, reconstruyendo a través de las palabras de

\footnotetext{
1 «En Francia, el Istitut Panos, en 2007, señaló la existencia de 240 programas de radio y 20 canales de televisión dirigidos a población migrante. Estados Unidos presenta una amplia constelación de medios de comunicación denominados 'étnicos'. En 2009, en el territorio estadounidense se contaban 3000 noticieros en 13 idiomas, con una audiencia estimada de 51 millones de ciudadanos» (Caritas-Migrantes, 2012: 213).

2 «Se trata de productos que forman parte de un sector más amplio, el de los medios de comunicación étnicos que, como afirma Dayan (1998), incluyen tanto los productos realizados por los inmigrantes para sus comunidades, como los productos realizados por parte de instituciones o asociaciones que actúan en el territorio que acoge a los inmigrantes» (Vittadini, 2007: 165).
} 
los entrevistados sus puntos de vista sobre el canal satelital, realizando, por lo tanto, un análisis narrativo de los contenidos.

Por último, se esbozarán una serie de reflexiones en torno a las conclusiones que se sacaron de esta investigación.

\section{Metodología}

La metodología que se ha utilizado para la realización de este estudio empírico ha sido de carácter cualitativo. Para nuestra reseña sobre la oferta televisiva se han monitoreado las programaciones italianas que a lo largo del tiempo han optado por invertir en la producción de programas televisivos diseñados para inmigrantes: un rápido excursus histórico que permitirá evaluar los progresos (o las regresiones) en la oferta de contenidos informativos, de entretenimiento y de servicio para los «nuevos italianos». Para esta reconstrucción se han utilizado artículos y trabajos que los mismos han analizado.

Nuestro análisis, empezando por los espacios que a lo largo del tiempo las emisoras públicas y privadas han reservado a los inmigrantes, se detendrá en un segundo momento en Babel TV, programación diseñada «a medida del inmigrante». En particular, se ilustrará la programación de la temporada televisiva 2013-2014, centrando la atención en las tres siguientes tipologías: series, informativos y cultura, y producciones originales (programas ideados y producidos por Babel TV). Para este artículo se han revisado las páginas en Internet de programas de Babel TV y algunas entrevistas audiovisuales a la directora del canal, Beatrice Coletti ${ }^{3}$.

Como trabajo previo de documentación, se ha realizado una búsqueda de fuentes bibliográficas que analicen los contenidos de programas de Babel TV y sus representaciones de los inmigrantes, así como de artículos de prensa que informen de la presentación pública y la desaparición de algunos de estos programas ${ }^{4}$.

También se analizaron los contenidos de las páginas web de los programas. Y por último, se visionaron decenas de capítulos de estos programas ${ }^{5}$ : en el caso de Babel TV, a través de su página web ${ }^{6}$.

\footnotetext{
3 Entrevistas encontradas en https://www.radioradicale.it/scheda/363283/babel-tv-la-prima-tv-dei-nuovi-italiani-intervista-abeatrice-colletti.

${ }^{4}$ En esta parte del trabajo sólo se indicarán los periódicos que se han interesado en la difusión de la programación de Babel TV. Se remite al texto de investigaciones previas para un estudio completo de las noticias que la prensa ha dedicado a los programas de este canal. Los periódicos en cuestión son: La Nazione, II Resto del Carlino, La Stampa, Avvenire y La Repubblica.

5 Hasta este punto, se observaron especialmente tres series televisivas disponibles en su página web: II secolo magnifico (EI magnífico siglo, Babel TV, 2013-2014. Origen: Turquía), Tre donne (Tres mujeres, Babel TV, 2012-2014. Origen: Siria), Una scommessa con la vita-Pariu cu viata (Una apuesta con la vida, Babel TV, 2012-2014. Origen: Rumania). Hemos seguido los tres primeros episodios de cada una de las series para empezar a comprender las estrategias de representación de este programa televisivo en particular. En cuanto a los programas de información (Babzine) y las producciones originales de Babel TV (Invito a cena, Kairos. L'attimo presente, G3 \& Piccole Mamme Crescono) se utilizaron algunos episodios disponibles sobre Youtube. Esta observación se realizó durante el mes de noviembre de 2017.

${ }^{6} \mathrm{http}: / /$ it-it.facebook.com/babeltv/.
} 
En cuanto al análisis del consumo televisivo por parte de los inmigrantes, la técnica de investigación que parece más apropiada para centrar los objetivos de la investigación ha sido la entrevista semi-estructurada.

Con respecto al contexto, la investigación se ha realizado en una ciudad de tamaño medio en el sur de Italia: Messina. La elección no sólo depende de las oportunidades procedentes del excelente conocimiento del territorio, sino que parece interesante investigar las dinámicas migratorias y los aspectos relacionados con las mismas en territorios no tan «atractivos» en términos económicos y sociales (Centorrino y David, 2009). Messina es una ciudad de 232.555 habitantes (Istat, 2018) y en enero de 2017 contaba con 17.631 ciudadanos extranjeros (ANPAL, 2017). Estos números nos informan de la considerable presencia de extranjeros en esta ciudad. Con respecto a las procedencias de los mismos, Asia es el continente de donde la mayoría de ellos es originario. El continente asiático, a su vez, está representado en muy alto grado por extranjeros procedentes del Sri Lanka, de Filipinas y de China.

El modelo de entrevista semi-estructurada ha sido elaborado y subdividido en tres partes. En la primera parte se esboza un perfil sociodemográfico de la persona. La segunda parte se propone analizar uso y consumo de la televisión. Las preguntas están encaminadas a determinar las predisposiciones en base a las elecciones televisivas y a las expectativas con respecto a la interacción con los productos mediáticos. La tercera parte, finalmente, tiene el objetivo de comprender la función social de la televisión: el significado que los telespectadores inmigrantes atribuyen a las experiencias de consumo mediático, reservando una atención particular a las percepciones relativas a la representación de la inmigración y a los procesos de integración en la pequeña pantalla.

Las directrices elaboradas para el desarrollo de estas entrevistas tienen 6 apartados: (1) datos personales del entrevistado; (2) contexto familiar y sus dinámicas; (3) proyecto migratorio (motivos de emigración, descripción de la salida del país, deseo de retorno en su país de origen); (4) vida cotidiana actual en Italia (actividades habituales, ocupación del tiempo libre en general, procesos de sociabilidad con migrantes/italianos y participación ciudadana); (5) uso y consumo de la televisión (los tiempos y las elecciones efectuadas con respecto al consumo televisivo); (6) la función social de la televisión (la relación entre el consumo televisivo de los sujetos de estudio y los imaginarios sociales de éstos) y la integración o formas de discriminación a través de la televisión (el efecto que las imágenes y el contenido de la televisión pueden tener en los discursos sobre la vida cotidiana de los inmigrantes, con especial referencia a las vías de integración o formas de discriminación).

La información necesaria para la realización de esta parte del trabajo se obtiene de 60 entrevistas (todas ellas se registraron electrónicamente) a 30 mujeres y a 30 hombres. Este trabajo de campo se realizó entre enero de 2017 y mayo de 2019. Se ha preservado el anonimato de las personas entrevistadas. Sin embargo, para facilitar el trabajo de análisis comparado, se ha asignado a cada entrevistado un código (de 01 a 10) combinado con la primera letra de cada nombre en mayúscula y con la nacionalidad.

Más específicamente, el perfil de los entrevistados se compone de la siguiente manera: 20 filipinos (diez mujeres y diez hombres), 20 ceilaneses (diez mujeres y diez hombres), 20 chinos 
(diez mujeres y diez hombres), de edad entre los $30 \mathrm{y}$ los 50 años puesto que los datos sobre el territorio (ANPAL, 2017; Salanitro, 2012), detectan la presencia más conspicua en esta franja de edad (31,96 por ciento de los extranjeros).

Para acceder a estos espacios sociales, se ha conseguido la cooperación de un determinado miembro de la comunidad, reconocido y respetado por todos. La selección de los entrevistados ha seguido un criterio intencional (Patton, 2002). Se trata de una muestra de carácter no estratificado, no probabilístico que, por lo tanto, no puede asegurar una representatividad estadística a los resultados obtenidos. De todas las maneras, era imposible lograr el mencionado objetivo de la representatividad estadística tanto, por el reducido número de los entrevistados, como por la esencia propia del medio de entrevista utilizado ${ }^{7}$.

\section{Los comienzos: la televisión pública para los inmigrantes}

Uno de los primeros intentos de crear una nueva narración de la inmigración, por lo que atañe a la historia de la televisión italiana, se produjo en 1988 gracias a una sección de la RAI(servicio público de radiodifusión en Italia) dedicada a los distintos aspectos de la inmigración en Italia, que se valía de la colaboración de periodistas italianos y extranjeros y que obtuvo un gran éxito de audiencia: Nonsolonero (Cava, 2011).

Esta sección del $\operatorname{Tg} 2^{8}$, de quince minutos de duración —emitida entre 1988 y 1993-, describía las condiciones sociales, culturales y económicas de los países de origen de los inmigrantes, así como la vida de los mismos en Italia, incluyendo su trabajo, familia y salud. Fue el primer programa en Italia dedicado a los temas de la inmigración y el racismo y el primero en proponer la conducción a una periodista caboverdiana, Maria de Lourdes Jesús, que se convertiría en punto de mediación entre las audiencias de italianos e inmigrantes.

La atención se enfocaba en el tratamiento de los derechos fundamentales de los extranjeros; por primera vez, en un espacio de los medios de comunicación de masas, se entreveía el punto de vista del migrante. Sin embargo, la transmisión fue suspendida de manera inesperada, aunque obtuvo una buena audiencia, al estar vinculada a un telediario con muchos espectadores.

El relato televisivo de las historias de inmigración continúa, en 1998, gracias a otro programa emitido por RAIdue: Un mondo a colori. Un programa de RAI Educational conducido por el periodista congolés Jean-Léonard Touadi primero (hasta 2004) y, posteriormente, por Valeria Coiante (autora y presentadora) que estaba dedicado al fenómeno de la inmigración y a los procesos de integración social en Italia. En este caso, se ponía el acento en la narración de las vivencias de inmigrantes en Italia que representaran casos ejemplares de integración. Por primera vez, los inmigrantes se presentaban como un recurso para la sociedad italiana. La propuesta de reportaje sobre la condición multiétnica de las ciudades italianas y

\footnotetext{
${ }^{7}$ El objetivo de este trabajo no es producir datos que puedan ser extrapolados a una población más amplia, de ahí el tipo de investigación utilizada. La intención, más bien, es la de conocer, a través de un estudio de caso concreto y de carácter cualitativo, sobre las experiencias, significados atribuidos e imaginarios sociales de un grupo de personas con experiencias migratorias diferentes.

8 Informativo de la segunda cadena pública italiana.
} 
sobre las leyes reguladoras de la inclusión de los inmigrantes se sumaba a documentales sobre los países de origen de los inmigrantes (Balma Tivola, 2001; Centro Studi e Ricerche Idos, 2012).

A partir de 1999, se emitió durante 12 años el semanario de RAI Shukran, dedicado también a los temas de la integración y de la inmigración, con la interesante decisión de la redacción, en la temporada 2009-2010, de abandonar el plató para que el programa se sumergiera en la vida cotidiana llevándolo — invitados incluidos — a la calle.

Lamentablemente, estos primeros y exitosos experimentos no dieron lugar a nuevas trayectorias narrativas y la representación de la inmigración no cambió de manera significativa (Faso, 2008; Gariglio, Pogliano y Zanini, 2010; Maneri, 2012).

En 2009, el programa Crash. Contatto, impatto, convivenza representa otro intento por parte de la televisión generalista de crear un espacio enteramente dedicado a los temas de la inmigración e integración.

Mediante reportajes filmados y entrevistas en el estudio se analizaba la intensidad del impacto del fenómeno migratorio en Italia. Los temas de la actualidad como clandestinidad, derechos civiles, integración racial formaban el corazón del programa.

Crash. Contatto, impatto, convivenza es un ejemplo de televisión de servicio público o, como afirma el director de RAI Educational, Giovanni Minoli (2009), un ejemplo de televisión donde las imágenes sustituyen las palabras que no tienen relación con la realidad.

A partir de 2011 en RAI $3^{9}$ se emitió el programa Radici. El otro lado de la migración, una serie de reportajes de Davide Demichelis. El objetivo se centraba en la vida cotidiana del inmigrante en Italia: su trabajo, su casa, la ciudad y la gente con la que vive, pero también cuánto de la cultura y de las tradiciones de su país él ha trasladado a Italia.

Cabe mencionar también RAI Med, el canal —emitido entre 2001 y 2014 - que RAI quiso crear para establecer un diálogo entre el mundo árabe e Italia, así como entre las numerosas comunidades de lengua árabe italianas y europeas.

Si pasamos a las emisoras privadas, resulta significativo sólo el episodio piloto del programa de información Barbari, emitido en 2007 en La7, con el objetivo de recoger testimonios e historias de los protagonistas de la inmigración. Pese a una buena acogida, el programa nunca vio la luz.

\section{La televisión por satélite: el caso de Babel TV}

Si dejamos de lado la oferta generalista y nos dirigimos a los guiones de la plataforma satelital, veremos un canal de televisión dedicado a los «nuevos inmigrantes»), es decir, los inmigrantes en Italia de primera y segunda generación: Babel TV, el canal de Sky — nacido en noviembre de 2010 para los inmigrantes presentes en Italia ${ }^{10}$.

\footnotetext{
${ }^{9}$ Tercera cadena pública italiana.

10 Otro ejemplo similar es Romit TV, canal para población rumana hecho en Italia.
} 
Se presentaba como una suerte de 'vademécum' para vivir bien en Italia, para conocer su idioma, leyes y mundo laboral; una iniciativa mediática que deseaba unir pueblos y culturas a través de programas de televisión.

Este canal de televisión se dedicaba a los 'nuevos italianos', tal como los definía la directora del canal, es decir, a las principales comunidades de extranjeros que viven en Italia y que representaban tanto la audiencia como los protagonistas del canal. Una 'ventana' abierta a las historias, a los usos y costumbres, a los rostros de las personas que viven en Italia.

Visto el carácter peculiar del canal, que también ha conseguido el premio Hot Bird 2011 en la sección Cultura y Educación (el premio europeo a los canales vía satélite), Babel TV puede considerarse un importante ejemplo internacional de medio televisivo capaz de abordar y tratar cuestiones importantes como la inmigración y la integración a través de la clave del entretenimiento televisivo.

Este canal tenía numerosos colaboradores externos y cuatro editores de origen extranjero, procedentes de los cuatro continentes de referencia (África, Asia, Europa oriental y Sudamérica).

Babel TV ofrecía a una audiencia de inmigrantes citas fijas y recurrentes que le daban mucho espacio a temas cívicos y sociales, cursos de italiano y ciudadanía, sugerencias sobre el mundo laboral en Italia; asimismo, era una herramienta para la participación individual y colectiva.

Lamentablemente, esta experiencia solo duró cuatro temporadas. Babel TV representa el mejor ejemplo de una parrilla de programación que cuenta la realidad de los inmigrantes a través de sus ojos y su voz y se convierte en un lugar en que las comunidades culturales pueden expresarse en el ámbito de la opinión pública. Por ello, ahora se pasará a describir la configuración de la parrilla de programación de la última temporada emitida (2013-2014), centrando la atención en las tres siguientes tipologías: series, informativos y cultura, y producciones originales (programas ideados y producidos por Babel TV).

En cuanto al entretenimiento, en particular, es preciso considerar el repertorio de series de televisión internacionales procedentes de las áreas del mundo en que Babel TV se centraba.

El objetivo era relatar el mundo de los nuevos italianos a través de las series de televisión adquiridas en sus países de origen, con la intención, también, de gratificarlos permitiéndoles ver en primera visión las últimas novedades en cuanto a series procedentes de América latina, Norte de África, Asia y Polonia.

Se trataba de series de gran éxito, escritas, producidas y emitidas en aquellos países. Viendo en Italia estas series en versión original con subtítulos, tanto los nativos de esos países como los italianos tenían la oportunidad de conocer algo más acerca de los usos, tradiciones de las personas de estos países y descubrir cómo son sus relaciones sentimentales y sociales. Esta tipología de programa permite a los televidentes volverse a encontrar consigo mismos, con su mundo social, estilos de vida, rostros y paisajes familiares (Cava, 2014).

Uno de los productos más interesantes en cuanto al género noticias era el magacín diario que permitía ver las noticias tanto en italiano como en los idiomas de las seis principales étnias 
presentes en Italia: BABzine ${ }^{11}$. Se trataba del programa de identificación del canal, que ofrecía cada día noticias sobre Italia a través de reportajes sobre la familia, el mundo laboral, el tiempo libre. Era un programa de información perfecto para explicar el beneficio de vivir en Italia, un espacio para recibir consejos útiles sobre las leyes y sobre todas las oportunidades de permanencia en Italia.

El resumen de prensa semanal de BABzine analizaba los artículos más interesantes publicados en los periódicos nacionales e internacionales con respecto a los temas de inmigración.

Los fines de semana, BABzine se convertía en la sección cultural BABzine CULT, dedicada a los eventos culturales; en particular, ayudaba a comprender que el mundo de los nuevos italianos es un mundo lleno de cultura. La sección daba a conocer músicos, pintores, escritores inmigrantes: todo un mundo que, una semana tras otra, se le ofrecía a los televidentes para que pudieran disfrutar de ese espacio cultural.

Respecto a las producciones originales, las más interesantes de la temporada de la que nos estamos ocupando son: Invito a cena (Invitación a comer, Babel TV, 2011-2012); Kairos. L'attimo presente (Kairos. El momento presente, Babel TV, 2013-2014); G3 ${ }^{12}$ \& Piccole Mamme Crescono (G3 y pequeñas mamás crecen, Babel TV, 2012-2014).

Invito a cena era un formato original que relataba, región por región, el encuentro entre un italiano y un extranjero que no se conocían y se encontraban por primera vez en una cena. $\mathrm{El}$ extranjero cocinaba un plato típico de su país para el italiano: una manera de hablar de los cambios que Italia estaba experimentando, así como del encuentro gracias a la comida, que siempre es un momento importante para establecer un diálogo.

Otra producción original de este canal de televisión fue Kairos. L'attimo presente, una serie documental escrita y dirigida por Davide Musicco, quien guiaba al espectador a través de las historias de inmigración de dos personas, con resultados opuestos. Por un lado, los que han conseguido integrarse, han formado una familia y construido una carrera laboral; por otro lado, quien ha intentado integrarse siendo rechazado por una sociedad compleja (y a menudo intolerante) como la italiana. La serie contaba las vivencias de inmigrantes procedentes de cualquier lugar del mundo en Roma y Milán, metidos en su vida cotidiana de extranjeros en dos grandes ciudades.

Se trataba de un programa sin filtros, cuyos protagonistas contaban su historia de manera realista. La narración se articulaba, por lo tanto, gracias a las palabras de los propios protagonistas, y seguía dos líneas paralelas, en una doble perspectiva de contraste y diálogo.

Finalmente, G3 \& Piccole Mamme Crescono, un docu-reality producido enteramente por mujeres e inspirado en «Piccole Mamme», el informe de Save the Children (2011) sobre madres adolescentes en Italia, que afrontaba, por primera vez, el tema del embarazo en edad adolescente entre las «nuevas italianas»».

\footnotetext{
${ }^{11}$ Realizado con la colaboración de Stranieri in Italia (el portal de Internet para los inmigrantes en Italia).

${ }^{12}$ Esta sigla en el título del programa se refiere al proceso de crecimiento de los niños, inmigrantes de tercera generación.
} 
En 2008 las «madres antes de tiempo» en Italia eran más de 10.000, el 18 por ciento de ellas de origen no italiano: una tasa cuantitativamente reducida que, sin embargo, merece especial atención debido a la red de personas a las que afecta - hijos, madres, padres, las familias de los padres - y a las causas que originan el fenómeno (Save the Children, 2011). Partiendo del estudio de esta estadística, G3 \& Piccole Mamme Crescono quería dar un rostro y una voz a algunas de estas jóvenes relatando el contexto en que se producían sus elecciones de maternidad.

En cada episodio se contaba la historia de una niña madre, cuya decisión de llevar a cabo el embarazo había sido influida por su propia experiencia de migración o por la de sus padres. Cada episodio empezaba cuando el embarazo y el nacimiento del niño ya se habían producido y las jóvenes madres vivían la experiencia del cuidado y de la crianza del pequeño. Se entraba en contacto con todas las figuras fundamentales de la vida de las niñas madres: el padre del bebé y su familia, la familia de la jóven, los amigos, profesores, empleadores, asistentes sociales y médicos.

El programa de Babel TV — G3 \& Piccole Mamme Crescono - reflejaba de manera cabal un escenario de maternidad complejo, centrándose en la jóven madre de origen extranjero que, además de las dificultades de ser una mamá adolescente, debía, lamentablemente, afrontar también problemas de integración en Italia.

\section{Análisis de datos: el consumo televisivo de Babel TV}

Para analizar el consumo televisivo de Babel TV a través de las entrevistas se buscó dar respuesta, principalmente, a las siguientes preguntas: ¿Conoces el canal satelital Babel TV? ¿Cuáles son los programas que te gustan más dentro de la parrilla de programación? ¿Por qué?

Podemos afirmar que tanto los hombres como las mujeres que han declarado conocer el canal han manifestado una elevada satisfacción en cuanto a los contenidos propuestos. En particular, lo que se desprende, como veremos, es que este espacio televisivo era considerado como espacio identitario. Por ejemplo, sobre eso, el entrevistado srilankese (05: T.) dice: «Ogni giorno con Babel TV imparavo qualcosa in più sull'Italia e questo mi aiutava a sentirmi italiano. Non solo conoscevo meglio lingua e abitudini di un popolo a me sconosciuto, ma iniziavo ad apprezzare l'atmosfera di questa terra! $\left.{ }^{13}\right\rangle$.

En general, en cuanto al consumo televisivo los hombres afirman ver la televisión por lo menos una hora al día, mientras las mujeres más de cuatro horas. Los hombres prefieren horarios de la tarde (desde las 19:00 horas); las mujeres se enfocan entre las 15:00 horas y las 23:00 horas.

En cuanto a la elección de los programas, los favoritos son programas de información, films y las producciones originales de Babel TV; en segundo lugar, las mujeres prefieren los programas de cocina y las series, los hombres prefieren los documentales.

\footnotetext{
${ }_{13}$ «Cada día con Babel TV aprendía algo más sobre Italia y esto me ayudaba a sentirme italiano. ¡No sólo conocía mejor idioma y costumbres de una población para mí desconocida, sino que empezaba a admirar la atmósfera de esta tierra!» (traducción de la autora).
} 
Acerca de las preferencias televisivas, por ejemplo, J., filipina (07) dice: «Le serie trasmesse su Babel TV permettevano ai telespettatori di ritrovare il proprio mondo, i paesaggi familiari ${ }^{14} \gg$. Resurgen, en definitiva, la sociedad y la cultura de pertenencia.

Las razones por las que les gustan estos programas son diferentes: algunos resultan favoritos porque les hacen reír o son divertidos. Por último, también aparecen respuestas como que cuentan historias que pasan en la vida.

Hablando de Babel TV, una mujer srilankesa (09: S.) dice: «Babel TV permetteva di far vedere gli immigrati come cittadini di primo ordine [...] non come una popolazione marginale, non come una invasione né come un problema [...]bensì come cittadini che meritano rispetto e diritto alla cittadinanza come chiunque ${ }^{15} \gg$.

En nuestro trabajo de campo, lo que se ha observado es que el seguimiento de algunos programas de Babel TV suele ser consecuencia del deseo de aprender la lengua del nuevo entorno de convivencia.

Todos los entrevistados indican Babzine, el magazín de información de Babel TV como el principal origen de conocimiento de los acontecimientos en el país de origen tanto como a la crónica italiana.

Por ejemplo, una mujer filipina (08:P.) afirma: «Vedevo le notizie del mio paese. La rassegna stampa settimanale analizzava gli articoli più interessanti pubblicati dai giornali internazionali $\left.{ }^{16}\right\rangle$.

Es interesante reconocer este deseo de información que destaca las palabras de los inmigrantes entrevistados y que parece no distinguir entre las noticias de la tierra de origen y la información del territorio de destino. Estas reflexiones nos revelan un sentido de pertenencia ambivalente: aunque predomine el vínculo a la familia y al territorio de origen, las personas entrevistadas muestran interés y deseo de aprender una manera de vida italiana. Y también predisponen positivamente al inmigrante para afrontar la vida cotidiana en Italia.

Asimismo, todos los entrevistados han mostrado un enorme interés por los programas de servicio del canal que tenían por objetivo orientar a los 'nuevos ciudadanos' en los ámbitos legales, administrativos y sanitarios italianos.

Erano programmi che davano voce alle nostre esigenze; si avevano orientamenti sul mondo del lavoro con rubriche sull'occupazione in Italia ${ }^{17}$ (41: C., china).

\footnotetext{
${ }^{14}$ «Las series trasmitidas en Babel TV permitían a los televidentes volver a encontrar su mundo, paisajes familiares» (traducción de la autora).

15 «Babel TV permitía visualizar a los inmigrantes como ciudadanos de primer orden [...], no como una población marginal, ni como una invasión ni como un problema [...], sino como ciudadanos que merecen respeto y derecho a la ciudadanía como otros cualquiera» (traducción de la autora).

${ }^{16}$ «Veía las noticias de mi país. El resumen de prensa semanal analizaba los artículos más interesantes publicados en los periódicos internacionales» (traducción de la autora).

17 «Eran programas que daban voz a nuestras exigencias; se encontraban recomendaciones sobre el mundo laboral con programas sobre el empleo en Italia».
} 
Los entrevistados señalan la diferencia entre como Babel TV representaba la imagen de los extranjeros y las narraciones que las emisoras televisivas italianas hacen del «Otroinmigrante».

Si los programas de Babel TV cuentan vidas de éxito, la normalidad de la vida de todos los días, la televisión italiana exaspera con tonos alarmantes. Los inmigrantes en el espacio medial italiano siempre aparecen a los márgenes de la sociedad. Babel TV en cambio presentaba a los inmigrantes como protagonistas activos en ambientes cotidianos, como un recurso para la sociedad italiana.

Esto es lo que dice un hombre srilankés (04: S.): «Quando accendo la TV gli immigrati sono sempre nei telegionali descritti come ladri o assassini oppure come disperati che annegano nel Mediterraneo. Mi manca vedere stranieri che vivono proprio come gli italiani e Babel TV faceva vedere questo ${ }^{18}$ ».

Y el entrevistado filipino (02: E.), asimismo, afirma: «Gli italiani hanno paura degli stranieri perchè in televisione si parla di loro con paura, Babel TV era l'unico canale che parlava degli stranieri come i programmi italiani parlano degli italiani ${ }^{19}{ }^{\prime}$.

En este tema, especialmente las mujeres, mencionan las producciones originales de Babel TV. La producción más popular es: Invito a cena. Las cocinas étnicas acercaban las diferentes comunidades favoreciendo encuentros serenos entre culturas. La comida en este programa se convierte en un momento importante para establecer un diálogo.

Sólo un entrevistado entre toda condena con firmeza la interrupción de la programación de Babel TV e interpreta esta cancelación como una acción discriminatoria: la interrupción del canal dedicado a la audiencia inmigrante refleja en sus propios ojos una clara voluntad de exclusión de este tipo de público.

\section{Conclusiones}

Ante todo, se precisa que nuestros análisis son preliminares, porque aún no se ha utilizado un programa de análisis de datos cualitativo. Lo que se ha desarrollado es un primer análisis realizado de manera 'manual' a partir de una lectura de los materiales producidos en el campo.

La metodología utilizada nos permitió dar respuesta a las preguntas y objetivos de investigación planteados. Igual que para la mayoría de los ciudadanos del país que los acoge, la televisión desempeña para los inmigrantes un papel importante en la organización del tiempo libre.

Los medios de comunicación entran, sin lugar a duda, en el debate sobre migraciones y desarrollan un papel significativo en la percepción del fenómeno migratorio, tanto en las sociedades de origen como en las de acogida (Etchegaray, Correa y Portales, 2015; Giusti, 2015).

\footnotetext{
${ }^{18}$ «Cuando enciendo la TV los inmigrantes siempre están en los noticiarios descritos como delincuentes o asesinos o como desesperados que se ahogan en el Mediterráneo. Me falta ver a extranjeros que viven exactamente como los italianos y Babel TV mostraba precisamente esto» (traducción de la autora).

19 «Los italianos le temen a los extranjeros porque en televisión se habla de ellos con terror, Babel TV ha sido el único canal que hablaba de los extranjeros como los programas italianos hablan de los italianos» (traducción de la autora).
} 
El conocimiento de la actitud de los inmigrantes con respecto a la televisión nos permite explicar qué tipo de uso se le otorga a este medio, y al mismo tiempo profundizar en el papel mediador que este medio de comunicación juega (Chavero y García - Muñoz, 2005: 201 y 202).

En esta fase de la investigación empírica, se ha observado la gran disponibilidad de las personas entrevistadas. Algunos han contestado lentamente, otros en forma rápida, pero con mucha motivación de contar los diferentes aspectos y necesidades de sus personalidades y sus identidades, viviendo dentro de una sociedad de acogida que, a distancia de muchos años de su llegada, aparece más consciente y dispuesta a relacionarse con la pluralidad de los mundos y de las expectativas de los inmigrantes. Nos han concedido su limitado tiempo libre, se reunieron con nosotros en sus casas con una taza de café, un té o una tisana y nos lo han contado.

De nuestra investigación destacan diferentes consideraciones. Del análisis efectuado hasta ahora, los inmigrantes parecen haber conseguido en Italia un buen nivel de integración: una buena comprensión de la lengua italiana, una buena satisfacción por los servicios sociales ofrecidos a los inmigrantes y una buena adhesión a los modelos de consumo, en particular en lo que se refiere a los productos tecnológicos.

Estas reflexiones tienen que considerar el hecho que dejarse entrevistar ya enseña una actitud abierta y proactiva hacia el proceso de integración. En este sentido, tenemos que señalar que algunos inmigrantes se negaron a formar parte de la investigación.

En algunas situaciones en las que nos aproximamos directamente a algunos 'hipotéticos' encuestados, pidiendo participar a la investigación, obtuvimos una negativa rotunda. Algunos de ellos argumentaron creer que su timidez no le hubiera permitido expresarse adecuadamente durante las entrevistas y otros más dijeron que sí, pero cuando quedamos para la primera entrevista, no se encontraron en su casa, o cancelaron el encuentro. Este rechazo es un hecho que no podemos ignorar y que nos proponemos analizar en una fase posterior de la investigación.

Volviendo a quienes han participado en nuestro proyecto, la televisión, medio poderoso y muy popular, desempeña actualmente tanto una función de entretenimiento, como un papel fundamental de socialización y contaminación con el estilo de vida de Italia.

El medio televisivo tiene gran aceptación en todos los inmigrantes entrevistados, quienes afirman que su consumo de televisión está supeditado a sus jornadas de trabajo. En las respuestas se aprecia una valoración positiva de la oferta que tienen a su disposición; en general, los inmigrantes entrevistados dan un voto más que bueno a la calidad de Babel TV. En nuestro trabajo de campo, lo que se ha observado es que el seguimiento de los programas suele ser, sobre todo, consecuencia del deseo de aprender la lengua del nuevo entorno de convivencia.

La información obtenida en esta investigación también revela una especial atención hacia los formatos de información y profundización en la misma. La televisión que nuestros inmigrantes utilizan es fundamental para el acceso a la información, al conocimiento y, al mismo tiempo, los mediatiza e influye sobre sus preferencias e intereses personales. En general, el consumo televisivo por parte de los inmigrantes responde a la necesidad de buscar información, entretenimiento (con series cómicas, comedias y animación) y/o aprender 
(especialmente aspectos sobre la cultura italiana). El consumo de TV satelital representaba, así, una relación directa con el país de origen, la comunidad étnica y la sociedad de destino. De esta manera — como puede leerse en la página web de Babel TV — «se animaba la convivencia y el conocimiento mutuo ${ }^{20} \gg$.

Babel TV ha sido una oportunidad extraordinaria para la experimentación, tanto en lo que se refiere a la actividad televisiva de adquisición de productos preparados de los mercados de los países que más representan las comunidades extranjeras en Italia - América del Sur, África del Norte, Europa Oriental y Asia - como también sobre la producción de programas originales y en el ámbito de la comunicación.

\section{Bibliografía}

AA.VV. (2012). Cosi vicine, cosi lontane: una ricerca sui bisogni e i consumi culturali delle cittadine straniere. Roma: Provincia di Roma.

AlliEVI, S. (2019). Immigrazione. Cambiare tutto. Roma-Bari: Laterza.

AltARRIBA, M. (2008). Usos i actituds dels immigrants davant dels mitjans de comunicación. Barcelona: Consell de l'Audiovisual de Catalunya (CAC).

ANPAL Servizi (2017). La presenza dei migranti nelle città metropolitane.

Recuperado de: https://www.anpalservizi.it/documents

BAGGIANI, B.; LONGONI, L. y SOLANO, G. (2011). Noi e l'altro? Materiali per l'analisi e la comprensione dei fenomeni migratori contemporanei. Ravenna: Discanti Editore.

BALMA TIVOLA, C. (2001). Colori della realtà. Immagini dell'immigrazione nei programmi di servizio RAI. Il nuovo spettatore, 5, 30-41.

BelluATI, M. (2015). Dalla fiction ai TG: la TV che piace agli immigrati. Problemi dell'informazione, XL (1), 245-248.

Binotto, M. y MARTino, V. (2004). FuoriLuogo. L'immigrazione e i media italiani. Cosenza: Pellegrini.

BINOTTO, M.; BRUNO, M. y LAI, V. (2016). Tracciare confini. L ’immigrazione nei media italiani. Milano: Franco Angeli.

BONERBA, G. (2013). Da uomo marginale a cittadino globale. Roma: Carocci Editore.

BRAMANTI, D. (2011). Generare luoghi di integrazione. Milano: Franco Angeli.

CALVANESE, E. (2011). Media e immigrazione tra stereotipi e pregiudizi. La rappresentazione dello straniero nel racconto giornalistico. Milano: Franco Angeli.

Caritas-Migrantes (2012). Dossier Statistico Immigrazione. $22^{\circ}$ Rapporto. Roma: Edizioni IDOS.

CAVA, A. (2011). Da Non solo Nero a Babel TV: una breve storia dell' «immigrato mediatico». En D. Carzo (Ed.). Narrare l'altro. Pratiche discorsive sull 'immigrazione (pp. 55-72). Roma: Aracne Editrice.

${ }^{20} \mathrm{http}: / /$ it-it.facebook.com/babeltv/ 
CAVA, A. (2014). Ombre migranti. Pratiche discorsive sullo straniero. En P. Musarò y P. Parmiggiani (Eds). Media e migrazioni. Etica, estetica e politica del discorso umanitario (pp. 157-170). Milano: Franco Angeli.

CARZO, D. (2011). Narrare l'altro. Pratiche discorsive sull'immigrazione. Roma: Aracne Editrice.

CARZO, D. (2016). Culture globali e Mediterranee. Migrazioni, Integrazioni, Noismi. Roma-Messina: Corisco.

Censis, (2002). Tuning into Diversity: immigrati e minoranze etniche nei media. Roma: Censis.

Centorrino, M. y DAVID, P. (2009). Le città della fata morgana. $5^{\circ}$ Rapporto sull'economia della provincia di Messina: analisi socio-economica e stili di vita nell'Area dello Stretto. Milano: Franco Angeli.

ChAVERO, H. y GARCíA-MuÑOZ, N. (2005). Los hábitos del consumo televisivo de una comunidad extranjera: el caso de estudiantes latinoamericanos en Barcelona. ZerRevista de estudios de comunicación, 19, 191-204.

CoRsinI, F. (2013). Raccontare l'altro: la rappresentazione delle diversità. En M. Buonanno (Ed.). Tempo di fiction. Il racconto televisivo in divenire (pp. 59-69). Napoli: Liguori.

EBerl, J.; MeltZer, C. E.; HeIDENREICH, T.; Herrero, B.; ThEORIN, N.; Lind, F.; BerGanZA, R.; BOOMGAARDEN. H. G.; SCHEMER. C. y STRÖMBÄCK, J. (2018). The European media discourse on immigration and its effects: A literature review. Annals of the International Communication Association, 1-17.

EtChegaray, N.; CorreA, T. y PORTAleS, D. (2015). Media consumption and immigration: Factors related to perception of stigmatization among immigrants. International Journal of Communication, 9, 3601-3620.

FASO, G. (2008). Lessico del razzismo democratico. Le parole che escludono. Roma: Derive Approdi.

GAdOTTI, G. (2009). Media e immigrati: un'analisi qualitativa. En L. Bovone y C. LunGHI (Eds). Consumi ai margini (pp. 171-204). Roma: Donzelli Editore.

Gariglio, L.; Pogliano, A. y ZANINI, R. (2010). Facce da straniero. 30 anni di fotografia e giornalismo sull 'immigrazione in Italia. Milano: Mondadori.

GIUSTI, M. (2015). Immigrazione e consumi culturali. Un'interpretazione pedagogica. Roma-Bari: Laterza.

GUADAGNUCCI, L. (2010). Parole sporche. Clandestini, nomadi, vu cumprà: il razzismo nei media e dentro di noi. Milano: Altreconomia.

Idos-Centro Studi e Ricerche (2012). Comunicare l'immigrazione. Guida pratica per gli operatori dell'informazione. Bologna: Litosei.

IgARTUA, J. J.; BARrios, I. M. y ORTEGA, F. (2012). Analysis of the Image of Immigration in Prime-Time Television Fiction. Comunicación y Sociedad, 2, 5-28.

ISTAT, (2008). L'uso dei media e del cellulare in Italia. Indagine multiscopo sulle famiglie "I cittadini e il tempo libero" (Anno 2006). Roma: Istat. 
KING, R. y WoOD, N. (2013). Media and migration. Constructions of Mobility and Difference. London and New York: Routledge.

MANERI, M. (2001). Il panico morale come dispositivo di trasformazione dell'insicurezza. Rassegna Italiana di Sociologia, XLII (1), 5-40.

MANERI, M. (2011). I media interculturali: una panoramica dettagliata. Libertà civili, 2, 21-28.

MANERI, M. (2012). L'immigrazione nei media. La traduzione di pratiche di controllo nel linguaggio in cui viviamo. Anuac, 1(1), 24-37.

marcos Ramos, M; GonzÁlez de Garay B. y Portillo Delgado, C. (2019). La representación de la inmigración en la ficción serial española contemporánea de prime time. Revista Latina de Comunicación Social, 74, 285 a 307.

MELI, A. (2011). Le tante voci della nuova Italia multiculturale. Libertà civili, 2, 29-35.

Minoli, G. (2009). Ecco Crash, l'impatto dell'immigrazione. Recuperado de: https://www.primaonline.it/2009/10/22/75593/tv-minoli-ecco-crash-limpattodellimmigrazione/

MuSARÒ, M. y PARMIGGIANI, P. (2014). Media e migrazioni. Milano: Franco Angeli.

NAVARRO, L. (2015). Televisión pública y diversidad cultural en España: el caso de los programas dirigidos a «inmigrantes». Migraciones, 38, 111-135.

PATTON, M. Q. (2002). Qualitative research and evaluation methods. Thousand Oaks, California: Sage Publications.

PEDROZA, G. (2004). La radio comercial en Monterrey. Apuntes para caracterizar una región. Anuario de Investigación de la Comunicación, XI, 131-150.

REPOLL, J. (2004). Los otros: Estudios de audiencia. Una observación etnográfica de una audiencia multicultural en su interacción con la TV.Zer, 16, 105-120.

SALANITRO, A. (2012). L'immigrazione a Messina. Ricerca sugli stranieri residenti. Messina: Anolf-Cisl.

Save the Children Italia Onlus (2011). Piccole mamme. Rapporto di Save the Children sulle mamme adolescenti in Italia. Recuperado de: https://www.savethechildren.it

SCIORTINO, G. (2015). È possibile misurare l'integrazione degli immigrati? Lo stato dell'arte (quaderno 63). Trento: Università degli studi di Trento.

VITTADINI, N. (2007). Gli ethnic media: tra produzione e consumo. Ikon-Forme e processi del comunicare, 54/55, 165-180.

VITTADINI, N. (2008). Diete di consumo culturale e migranti. Ikon-Forme e processi del comunicare, 56/57, 13-37.

Para citar este trabajo: Cava, G. (2019). Breve historia de la televisión italiana destinada a inmigrantes: el caso de Babel Tv. index.comunicación, 9(3), 59-74. 